\title{
Modelling of current and temperature effects on supercapacitors ageing. Part II: State-of-Health assessment
}

\author{
Dimitri Torregrossa*, Mario Paolone \\ Distributed Electrical System Laboratory, Swiss Federal Institute of Technology, Lausanne, Switzerland
}

\section{A R T I C L E I N F O}

\section{Article history:}

Available online $\mathrm{xxx}$

\section{Keywords:}

Supercapacitor

Ageing test

State-of-Health

Life endurance

Power cycling

Recovering phase

\begin{abstract}
A B S T R A C T
This second part of the paper proposes a model for predicting the State-of-Health ( $\mathrm{SOH}$ ) of electrochemical double layers supercapacitors (SCs) during combined high-pulsed power cycling and high-temperature life endurance stresses. The evolutions of the SC capacitance, $C_{\mathrm{SC}}$, during these specific types of stresses have been discussed in part I and, based on the phenomenological observations we made, we here predict the $C_{\mathrm{SC}}$ evolution by means of a dedicated model. The inputs of the proposed model are the SC delivered charge and the duration of the temperature stress above the rated one; the output is the $C_{\mathrm{SC}}$. The main virtue of the model is its capability to take into account the presence of the $C_{\mathrm{SC}}$ recovering and accelerated ageing that cannot be represented by other models available in the literature. The validation of the proposed SC ageing model is carried out by means of experimental results, other than those used to infer the model, obtained on a 365 F SC stressed by combined life-endurance and power-cycling stresses over a time window of 35 days.
\end{abstract}

(c) 2015 Elsevier Ltd. All rights reserved.

\section{Introduction}

The assessment of the State-of-Health ( $\mathrm{SOH}$ ) of electrochemical double layer supercapacitor (SC) is of utmost importance for the correct design and operation of these storage devices. Indeed, along with the correct ageing prediction, it allows to accurately evaluate the cost associated to the future use of the device. The evaluation of the $\mathrm{SOH}$ also allows for identifying temporary degradations and, consequently, avoid major failures. Refs. [1-5] have discussed the use of the SOH of these devices for predicting the remaining lifetime and to define optimal energy management strategies.

From a macroscopic point of view, the ageing process of a SC involves the fading of its performances associated to a decreased capability of storing (and delivering) a given amount of charge. In order to quantify the ageing process, a large part of the literature has adopted as an indicator the so-called SC equivalent series resistance (ESR) and/or the SC capacitance $\left(C_{\mathrm{SC}}\right)$.

The first part of this work has already investigated the fading of these parameters during Life Endurance (LE), Power Cycling (PC) and combined LE and PC stresses. Additionally, the phenomenology describing the associated ageing processes has been described too.

\footnotetext{
* Corresponding author. Fax: +41 216934662.

E-mail address: dimitri.torregrossa@epfl.ch (D. Torregrossa).
}

From the best of the Authors' knowledge, even if there is a considerable amount of works discussing the SC ageing (i.e [6-10]), the literature concerning the computation of the $\mathrm{SOH}$ of these devices is quite scarce (to date, only [11] discusses this specific topic on a quantitative basis). On the contrary, major efforts have been focused on the SOH assessment of lithium cells [11-13]. ${ }^{1}$ However, the SOH estimation methods proposed in [11-13] present important drawbacks related to their computational complexity and/or need of large number of sensing devices.

In order to reduce the requirements associated to these drawbacks and focus on the specific case of SCs, this paper proposes a $\mathrm{SOH}$ estimator characterised by the following peculiarities:

1) it requires a limited number of sensing devices since it just relies on the measurement of the cell voltage, current and temperature;

2) it relies on a set of measurements that can be off-line performed on a sample SC cell;

3 ) it takes into account the effect of stresses associated to highpulsed delivered current and, also, high temperatures;

\footnotetext{
These works might be of interest for the SOH assessment of SC's in view of the common ageing process between these two electrochemical storage systems (e.g., effects of current on the electrode, effects of temperature on the viscosity and conductivity of the electrolyte).
} 
4) it takes into account temporary performance changes due to the $\mathrm{SC}$ recovering phases (RPs) and accelerated ageing phases (APs) $[14,15]$.

The part II of this work is structured as follows. Section 2 describes the elements of the physical process driving the ageing mechanism of a SC and that have been used to define the proposed $\mathrm{SOH}$ model. In this respect, a least square fitting is used to determine the parameters of the proposed $\mathrm{SOH}$ model during reference LE and PC stresses. The obtained SOH models are, then, integrated for predicting the $C_{\mathrm{SC}}$ evolution during combined PC and LE stresses. Section 3 illustrates how to modify the inputs of this look-up tables when the stress conditions differ from the reference one. We show that the obtained $\mathrm{SOH}$ model is capable to represent the accelerated ageing and recovering phases in a correct way. In this respect, a comprehensive experimental validation is illustrated in Section 4. In this section we made reference to a $365 \mathrm{~F} \mathrm{SC}$ exposed to combined LE and PC stresses other than those used to infer the SOH model. In this section we also discuss the mismatch that we would obtain in case AP and RP are not accounted. The last section summarizes the main contributions and outlooks the methodology here discussed.

\section{SOH mapping with respect to reference LE and PC stress conditions}

The part I of the work has described the ageing processes driving the performances fading of SCs during PC, LE and stresses combining high-pulsed PC and high-temperature LE. The description of the main ageing mechanism has been presented in order to physically explain the trends of the $C_{\mathrm{SC}}$ and ESR experimentally observed during the considered types of stresses. It is possible to summarize the main findings as follows:

(a) during a LE stress, the average SC temperature $\left(T_{\mathrm{SC}}\right)$ and the time duration $\tau_{\mathrm{D}}$ of the test (at $T_{\mathrm{SC}}$ ) are the main ageing factors;

(b) during a combined LE and PC stress, the $T_{\mathrm{SC}}$ and delivered charge $(A h)$ are the main ageing factors.

As known, ageing tests on electrochemical storage systems are usually time-consuming activities since the goal is to estimate the conditions for which the $C_{\mathrm{SC}}$ decreases to (typically) $80 \%$ of its initial value. An alternative to the brute-force testing to assess the ageing of the SC is to extrapolate the $C_{\mathrm{SC}}$ trend by means of a data fitting performed on a limited data set of a representative given stressing condition.

Two data fitting of the $C_{\mathrm{SC}}$ trends have been inferred for each type of the considered stresses: the first one links the $C_{\mathrm{SC}}$ (at constant temperature) and $\tau_{\mathrm{D}}$ for the LE stress, and a second one linking the $C_{\mathrm{SC}}$ with the SC delivered charge (at constant temperature) for the PC stress.

The following subsections describe the criteria used for the fitting equations for each type of test.

\subsection{Life endurance ageing}

In the first part of the work, it has been already illustrated that during LE stresses, the main reason of the $C_{\mathrm{SC}}$ degradation are the changes in: (i) the viscosity and in the conductivity of the electrolyte and; (ii) the electrode porosity. These changes are mainly due to the impurities into the electrolyte generated during its manufacturing and those produced during the SC usage.

It has already illustrated that the degradation of the conductivity and viscosity of the electrolyte vs the temperature are described by exponential functions (e.g., [17-20]). In this respect, part I has experimentally observed that the associated degradation process
Table 1

Summary of the inferred parameters of Eq. (1) for the mapping of the LE ageing.

\begin{tabular}{ll}
\hline Parameter & Value \\
\hline A & 29.35 \\
a & $17.5 \times 10^{-6}$ \\
B & 13.06 \\
b & $27.1 \times 10^{-6}$ \\
$C_{\mathrm{LE} \infty}$ & $307 \mathrm{~F}$ \\
\hline
\end{tabular}

of the $C_{\mathrm{SC}}$ is represented by two time constants. In the first $150 \mathrm{~h}$ of LE stress, it is possible to observe a quite fast $C_{\mathrm{SC}}$ degradation of $7 \%$. Then, for the next $1000 \mathrm{~h}$, the $C_{\mathrm{SC}}$ degradation is of $3 \%$ and, finally, it evolves towards a quasi-linear time trend. Indeed, both the impurities production and their active filling of the electrode pores are more important at the beginning of the stress. Then, they become relatively less important during the SC usage.

Additionally, it is worth noting that once the quasi-linear $C_{\mathrm{SC}}$ degradation is reached, the $C_{\mathrm{SC}}$ will tend to an asymptotic value depending on the type of stress. This value is in the range of 70-80\% of the starting $C_{\mathrm{SC}}$ if the $V_{\mathrm{SC}}$ and the $T_{\mathrm{SC}}$ are within the rated values. ${ }^{2}$

For the above reasons, the model adopted for mapping the $C_{S C}$ degradation evolution during a LE is composed by two exponential functions and a constant value (see Eq. (1)).

The $C_{\mathrm{SC}}$ evolution, ${ }^{3}$ called $C_{\mathrm{SC}}^{*}$, is then estimated during a LE stress test using the following model:

$C_{\mathrm{SC}, \mathrm{LE}}^{*}\left(\tau_{\mathrm{D}}\right)=A \mathrm{e}^{-a \tau_{\mathrm{D}}(t)}+B \mathrm{e}^{-b \tau_{\mathrm{D}}(t)}+C_{\mathrm{LE} \propto}$

where:

- $A \mathrm{e}^{-a \tau_{\mathrm{D}^{(t)}}}, B \mathrm{e}^{-b \tau_{\mathrm{D}^{(t)}}}$, are the two exponential functions taking into account the two time constants of the LE ageing process evolution before described.

- $C_{\mathrm{LE} \infty}$ is the value of the $C_{\mathrm{SC}}$ defined by the $\mathrm{SC}$ manufactures as the life-time expectation of the device.

The following least square fitting problem has been defined for inferring the above model parameters:

$\underset{A, a, B, b, C_{\mathrm{SC} \infty}}{\operatorname{argmin}}\left\{C_{\mathrm{SCM}}(t)-A \mathrm{e}^{-a \tau_{\mathrm{D}^{(t)}}}-B \mathrm{e}^{-b \tau_{\mathrm{D}^{(t)}}}-C_{\mathrm{LE} \infty}\right\}^{2}$

where $C_{\mathrm{SCM}}(t)$ is the measured $C_{\mathrm{SC}}$ each $24 \mathrm{~h}$.

The parameters that have been inferred from Eq. (1) are reported in Table 1.

The blue curve in Fig. 1 shows the measured evolution of the $C_{\mathrm{SC}}$ during a LE test performed for 60 days at $328.15 \mathrm{~K}$. The $C_{\mathrm{SC}}$ has been evaluated each $24 \mathrm{~h}$ by the experimental procedure described in [19]. The red curve shows the $C_{\mathrm{SC}}^{*}$ using the above model. The obtained root mean square error is equal to 0.997 .

\subsection{Power cycling ageing}

In part I, we have already discussed the fact that the main mechanisms driving the $C_{\mathrm{SC}}$ degradation during a PC cycling are similar the ones associated to the LE stress (i.e., viscosity and conductivity of the electrolyte and electrode porosity). The main difference between LE and PC stress is that the latter amplifies the degradation of the electrolyte and the production of impurities since it is associated to the current extraction (the so-called

${ }^{2}$ If the boiling point of the electrolyte $\left(78^{\circ} \mathrm{C}\right)$ and/or its decomposition voltage $(3.5 \mathrm{~V})$ are reached, other degradations, that are out of scope of the work here presented, take place and involve more important $C_{\mathrm{SC}}$ fading [15].

${ }^{3}$ We remind that this fitting refers to a LE stress obtained at constant average $T_{\mathrm{SC}}$ 


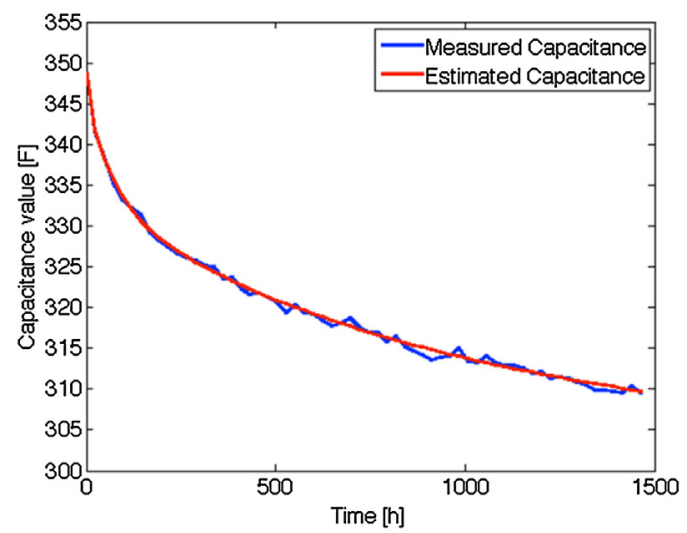

Fig. 1. Comparison between the measured $C_{\mathrm{SC}}$ evolution and the fitted one using Eq. (3) during a LE test at $328.15 \mathrm{~K}$. The results for each type of test report the average $C_{S C}$ obtained on the four SC samples under test.(For interpretation of the references to colour in the text, the reader is referred to the web version of this article).

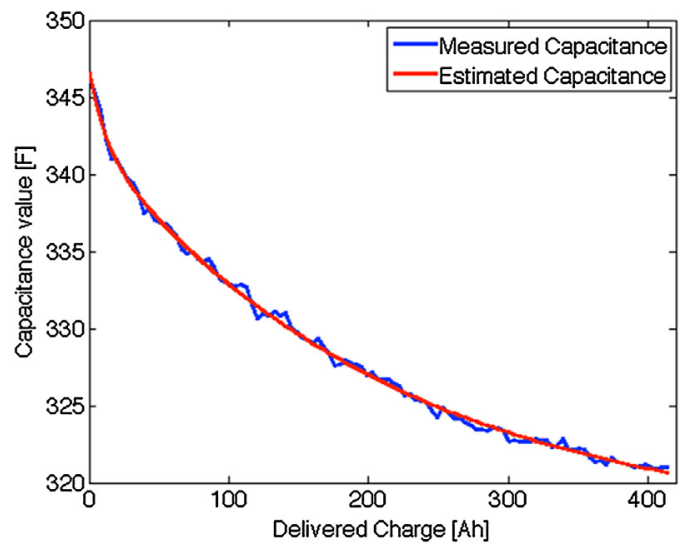

Fig. 2. Comparison between the measured $C_{S C}$ evolution and the fitted one by Eq. (3) during a PC test at $328.15 \mathrm{~K}$. The results for each type of test report the average $\mathrm{C}_{\mathrm{SC}}$ obtained on the four SC samples under test.(For interpretation of the references to colour in the text, the reader is referred to the web version of this article).

electrodes cracking).

Even for a PC stress, it has been experimentally verified that the $C_{\text {sc }}$ fading has two main times constant. For this reason, and those already described in the previous subsection, the model adopted for the mapping the $C_{\mathrm{sC}}$ evolution during a PC stress is composed by two exponential functions and a constant value (see Eq. (3)).

The blue curve of Fig. 2 illustrates the measured evolution of the $C_{S C}$ during a PC stress performed for 30 days at $328.15 \mathrm{~K}$. The red curve shows the estimated $C_{\mathrm{SC}}^{*}$, during a PC stress test, using the following model:

$C_{\mathrm{SC}, \mathrm{PC}}^{*}(t)=F \mathrm{e}^{-f A h(t)}+K \mathrm{e}^{-k A h(t)}+C_{\mathrm{PC} \infty}$

Table 2

Summary of the inferred parameters of Eq. (3) for the mapping of the PC ageing.

\begin{tabular}{ll}
\hline Parameter & Value \\
\hline $\mathrm{F}$ & 28.8 \\
$\mathrm{f}$ & $4.37 \times 10^{-3}$ \\
$\mathrm{~K}$ & 9.2 \\
$\mathrm{k}$ & $29.7 \times 10^{-3}$ \\
$C_{\mathrm{PC} \infty}$ & $302.6 \mathrm{~F}$ \\
\hline
\end{tabular}

where:

- $F \mathrm{e}^{-f A h(t)} \mathrm{Ke}^{-k A h(t)}$, are the two exponential functions associated to the PC ageing evolution above described.

- $C_{\mathrm{PC} \infty}$ is the value of the $C_{\mathrm{SC}}$ associated with the life-time expectation.

Also in this case, a least square fitting identical to Eq. (2) has been used to infer the unknown parameters of Eq. (3). They obtained parameters are reported in Table $2 .^{4}$

The blue curve of Fig. 2 illustrates the measured evolution of the $C_{\mathrm{SC}}$ during a PC stress performed for 30 days at $328.15 \mathrm{~K}$. The red curve shows the estimated $C_{\mathrm{SC}}^{*}$ using Eq. (3).

The root mean square error of the model proposed by Eq. (3) is of 0.996 .

\section{State-of-Health assessment during combined LE and PC stress test}

\subsection{General overview}

The mapping of the $C_{\mathrm{SC}}$ provided by Eqs. (1) and (3) has been obtained for separated LE and PC stresses. These can be considered as reference conditions. It is worth observing that during a real utilisation, SCs are not stressed with distinguished LE and PC stresses since they are superposed with different amplitudes and durations. We summarize here the main reasons for which Eqs. (1) and (3) cannot be used in a straightforward manner for the $C_{\mathrm{SC}}$ assessment during combined LE and PC stresses.

1. The initial value of the $C_{\mathrm{SC}}$ is not the same for all the SCs belonging to the same batch production. Since this value is accounted during the least square fitting described in Section 2.1, Eqs. (1) and (3) cannot directly assess the $C_{\mathrm{SC}}$ fading. Therefore, the prediction provided from Eqs. (1) and (3) has to be rescaled (for further details, see Section 3.2)

2. During a normal usage of a SC, in the transition from LE and PC, and vice versa, the phenomena described in $[14,15]$ will take place. The effects of these phenomena are a temporary fast $C_{\mathrm{SC}}$ fading during the transition $\mathrm{PC} \rightarrow \mathrm{LE}(\mathrm{AP})$ and a temporary increase of the $C_{\mathrm{SC}}$ during the transition $\mathrm{LE} \rightarrow \mathrm{PC}(\mathrm{RP})$.

3. Once the effects of the AP and RP on the $C_{\mathrm{SC}}$ have been accounted, there is the need to re-initialize the value of the inputs of the $\mathrm{SOH}$ model (i.e., $\tau_{\mathrm{D}}(t)$ for a LE stress and $A h(t)$ for a PC stress).

In view of the above, the main idea of this section is to use the models developed separately for the LE and PC stresses for a combined PC and LE stress other than the reference ones. The first step in this direction is to define the $\mathrm{SOH}$. We have used the following definition:

$\operatorname{SOH}(t)=\frac{C_{\mathrm{SC}}(t)}{C_{N}}$

where it is important to underline that $C_{N}$ is not the value declared by the SC manufacturer since, during the first hours of the SC cycling, there is already a decrease of $C_{\mathrm{SC}}$ not associated to any ageing phenomena but mainly related to the redistribution of the residual charge. ${ }^{5}$ In order to mitigate/eliminate this bias, we

\footnotetext{
${ }^{4}$ From Table 2 we can see that the inferred value of the $C_{\mathrm{PC} \infty}$ is of $302.6 \mathrm{~F}$. Also this value can be considered reasonable since is lower than the corresponding one after a LE test (it has been already underlined that during a PC stress test the ageing phenomena are amplified compared to those during a LE stress test).

Further details concerning this aspect can be found in [16].
} 
decided to select $C_{N}$ as the averaged value of the capacitance (obtained on 4 SC samples) and measured in correspondence of their 5th initial cycle.

\subsection{SOH model initialization}

As already anticipated, the value of rated capacitance is not the same for all the SCs belonging to the same batch production since it depends on several non controllable factors (i.e., those associated to the batch production and the ageing process that took place during the transportation and storing).

In this respect, it is important to scale the evolutions described by Eqs. (1) and (3) in p.u. of $C_{N}$. This value has to be measured at the beginning of the applied stress.

Let us define $C_{N}^{*}$ as the $C_{N}$ of the reference LE or PC stress, and $C_{N}^{i}$ the $C_{N}$ of a generic SC. Then, the scale factor to be applied to the result of Eq. (1) or (3) is simply $C_{N}^{i} / C_{N}^{*}$. In what follows, with the symbol $C_{\mathrm{SC}}^{*}$ we will refer to the $C_{\mathrm{SC}}^{*}$ value already scaled.

\subsection{Modelling of AP and RP effects}

Before illustrating the structure of the proposed $\mathrm{SOH}$ model, we summarize the main findings of [15] since we are interested to model the RP and AP.

a) The RP always takes place during the transition LE $\rightarrow$ PC. Its duration depends to the $T_{\mathrm{SC}}$ and on the time duration of the previous LE sub-phase. We can assume that the $C_{\mathrm{SC}}$ increase, at $323.15 \mathrm{~K}$, is of $0.95 \%$ of the $C_{\mathrm{SC}}$ value at the beginning of the transition. The recovering phase holds for $7 \mathrm{~h}$ if the previous $\mathrm{LE}$ sub-phase held, at least, for $24 \mathrm{~h}$.

b) The AP phase always takes place during the transition $\mathrm{PC} \rightarrow$ LE. Its duration depends on the $T_{\mathrm{SC}}$ and on the charge delivered before the transition. We can assume that the $C_{\mathrm{SC}}$ decrease, at $323.15 \mathrm{~K}$, is of $1.5 \%$ of the $C_{\mathrm{SC}}$ value at the beginning of the transition. The accelerated ageing phase holds for $12 \mathrm{~h}$ if the previous PC sub phase held for at least $12 \mathrm{~h}$.

Fig. 3 schematically illustrates the whole model for predicting the $C_{\mathrm{SC}}$ and, consequently, its SOH during: (i) separated LE and PC stresses other than the reference ones; (ii) combined LE and PC stress with AP and RP.

The main inputs of our $\mathrm{SOH}$ model are:

- the charge,Ah(t), delivered by the SC from the beginning of the test until time $t$ (if the SC is aged with a PC stress). ${ }^{6}$

- $\tau_{\mathrm{D}}(t)$, the time duration of the LE at $T_{\mathrm{SC}}$ since the beginning of the test until time $t$ (if the SC is aged with a LE stress).

Then, if the targeted SC is stressed with a combined LE and PC test, there are two additional sub-inputs:

- $\Delta A h$ : virtual negative quantity of charge (in Ah) to be added to $A h(t)$. It allows, along with Eq. (3), to take into account the increased value of $C_{\mathrm{SC}}$ after the recovering phase associated to the transition from LE toward PC stresses.

$\bullet$

\footnotetext{
${ }^{6}$ We neglect the charge delivered by the SC during a LE stress test for its experimental $C_{\mathrm{SC}}$ evaluation and consequently we assumed that idela LE operating condition are not violated. The RMS current during the assessment procedure is of 1.5 during $20 \mathrm{~min}$.
}

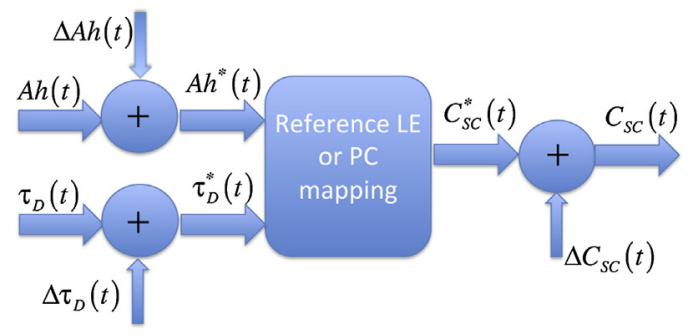

Fig. 3. schematic representation of the proposed $\mathrm{C}_{\mathrm{SC}}$ computation for the $\mathrm{SOH}$ prediction.

$\Delta \tau_{\mathrm{D}}$ : virtual time duration to be added to $\tau_{\mathrm{D}}(t)$. It allows, along with Eq. (1), to take into account the decreased $C_{\mathrm{SC}}$ after the accelerated ageing phase associated to the transition from PC test toward LE stresses.

The computation of both additional sub-inputs is described in the following subsections.

By observing Fig. 3, the value of $C_{\mathrm{SC}}^{*}(t)$ is then corrected by the following:

$C_{\mathrm{SC}}(t)=C_{\mathrm{SC}}^{*}(t)+\Delta C_{\mathrm{SC}}(t)$

where $\Delta C_{\mathrm{SC}}(t)$ is an additional virtual $\Delta C_{\mathrm{SC}}$ for taking into account the evolution of $C_{\mathrm{SC}}$ during the transition $\mathrm{LE} \rightarrow \mathrm{PC}$ test and vice versa.

The function $\Delta C_{\mathrm{SC}}(t)$, obtained from the results presented in [15], are described by Eqs. (4)-(7). ${ }^{7}$

For a transition $\mathrm{PC} \rightarrow$ LE test:

$\Delta C_{\mathrm{SC}}(t)=-0.45 \frac{C_{\mathrm{SC}}^{*}(t)}{100}$ at $t=3$ hours

$\Delta C_{\mathrm{SC}}(t)=-0.75 \frac{C_{\mathrm{SC}}^{*}\left(t_{0}\right)}{100}$ at $t=12$ hours

For a transition $\mathrm{LE} \rightarrow \mathrm{PC}$ test:

$\Delta C_{\mathrm{SC}}(t)=0.65 \frac{C_{\mathrm{SC}}^{*}\left(t_{0}\right)}{100}$ at $t=3$ hours

$\Delta C_{\mathrm{SC}}(t)=0.9 \frac{C_{\mathrm{SC}}^{*}\left(t_{0}\right)}{100}$ at $t=7$ hours

where $t_{0}$ is the starting time of the targeted transition and $C_{\mathrm{SC}}^{*}\left(t_{0}\right)$ the associated computed value of $C_{\mathrm{SC}}$.

\subsection{Modelling of the AP}

In order to quantify the effect of the accelerated ageing phase, we define the following quantities:

- $t_{\mathrm{AP}}$ : the time associated to the end of the accelerated ageing phase;

- $C_{\mathrm{SC}}\left(t_{\mathrm{AP}}\right)$ : the computed value of $C_{\mathrm{SC}}$ at the end of the accelerated ageing phase.

During this specific transition, in the model shown in Fig. 3 both

\footnotetext{
The results presented in [15] have been obtained from experimental investigations on a limited number of SCs and for a limited number of transitions allowing for AP and RP. We here claim that the effects of AP and RP could be considered of the same entity for all SCS of the same manufacturer. The
} experimental validation described in section IV support this hypothesis. 
inputs $A h(t)$ and $\Delta A h(t)$ are equal to zero (there is no charge delivered by the $S C$ ).

Since the main effect of AP is a temporary fast $C_{\mathrm{SC}}$ fading and Eq. (1) works only during a reference LE stress, the following equations are always satisfied:

$\tau_{\mathrm{D}}^{*}\left(t_{\mathrm{AP}}\right) \neq \tau_{\mathrm{D}}\left(t_{0}\right)+t_{\mathrm{AP}}$

$\tau_{\mathrm{D}}^{*}\left(t_{\mathrm{AP}}\right)=\tau_{\mathrm{D}}\left(t_{\mathrm{AP}}\right)+\Delta \tau_{\mathrm{D}}=\tau_{\mathrm{D}}\left(t_{0}\right)+t_{\mathrm{AP}}+\Delta \tau_{\mathrm{D}}$

$C_{\mathrm{SC}}^{*}\left(t_{0}\right)>C_{\mathrm{SC}}^{*}\left(t_{\mathrm{AP}}\right)$

$C_{\mathrm{SC}}^{*}\left(t_{\mathrm{AP}}\right) \neq A \mathrm{e}^{-\tau_{\mathrm{D}}\left(t_{\mathrm{AP}}\right)}+B \mathrm{e}^{-\tau_{\mathrm{D}}\left(t_{\mathrm{AP}}\right)}+C_{\mathrm{LE} \infty}$

Eqs. (6) and (7) allow for correcting the $C_{\mathrm{SC}}^{*}(t)$ during the transition from PC to LE stresses. Then, in order to properly compute the evolution of $C_{\mathrm{SC}}$ (for $t>t_{\mathrm{AP}}$ ) it is necessary to compute $\tau_{\mathrm{D}}^{*}\left(t_{\mathrm{AP}}\right)$ and, indirectly, $\Delta \tau_{\mathrm{D}}$.

Based on what already described, the set of equations allowing for the $C_{\mathrm{SC}}$ computation is summarized below:

$C_{\mathrm{SC}}\left(t_{\mathrm{AP}}\right)=C_{\mathrm{SC}}^{*}\left(t_{0}\right)+\Delta C_{\mathrm{SC}}\left(t_{\mathrm{AP}}\right) t_{0}<t<t_{\mathrm{AP}}$

$C_{\mathrm{SC}}^{*}(t)=A \mathrm{e}^{-a\left(\tau_{\mathrm{D}}^{*}\left(t_{\mathrm{AP}}\right)+\tau_{\mathrm{D}}(t)\right)}+B \mathrm{e}^{-b\left(\tau_{\mathrm{D}}^{*}\left(t_{\mathrm{AP}}\right)+\tau_{\mathrm{D}}(t)\right)}+C_{\mathrm{LE} \infty} t>t_{\mathrm{AP}}$

$\tau_{\mathrm{D}}^{*}\left(t_{\mathrm{AP}}\right) \rightarrow A \mathrm{e}^{-a \tau_{\mathrm{D}}^{*}\left(t_{\mathrm{AP}}\right)}+B \mathrm{e}^{-b \tau_{\mathrm{D}}^{*}\left(t_{\mathrm{AP}}\right)}+C_{\mathrm{LE} \infty}=C_{\mathrm{SC}}\left(t_{\mathrm{AP}}\right)$

The value of $\tau_{\mathrm{D}}^{*}$ satisfying Eq. (16) is computed by inverting Eq. (1). ${ }^{8}$

\subsection{Modelling of the RP}

Similar to subsection 3.5, to derive the mathematical model taking into account the effects of the recovering phase, we henceforth defined:

- $t_{\mathrm{RC}}$ the time associated to the end of the recovering phase;

- $C_{\mathrm{SC}}\left(t_{\mathrm{RC}}\right)$ the computed value of $C_{\mathrm{SC}}$ at the end of the recovering phase;

During this specific transition, in the model shown in Fig. 3 both inputs $\tau_{\mathrm{D}}(t)$ and $\Delta \tau_{\mathrm{D}}(t)$ are equal to zero (the ageing factor is the delivered charge).

Since the main effect of RP is a temporary increase of $C_{\mathrm{SC}}$ and, since Eq. (3) works properly only during a reference PC stress, the following equations are always satisfied:

$A h\left(t_{\mathrm{RC}}\right)=A h\left(t_{0}\right)$

$A h^{*}\left(t_{\mathrm{AP}}\right)=A h\left(t_{0}\right)+\Delta A h\left(t_{\mathrm{AP}}\right)$

$C_{\mathrm{SC}}^{*}\left(t_{0}\right)<C_{\mathrm{SC}}^{*}\left(t_{\mathrm{RP}}\right)$

$C_{\mathrm{SC}}^{*}\left(t_{R P}\right) \neq F \mathrm{e}^{-f A h\left(t_{\mathrm{RP}}\right)}+K \mathrm{e}^{-k A h\left(t_{\mathrm{RP}}\right)}+C_{\mathrm{PC} \propto}$

Eqs. (8) and (9) allow for evaluating the $C_{\mathrm{SC}}$ value during the transition from LE to PC stress. Then, in order to properly compute

\footnotetext{
${ }^{8}$ Note that is reasonable to assume that Eqs. (1) and (3) are bijective functions.
}

the evolution of $C_{\mathrm{SC}}$ (for $t>t_{\mathrm{RP}}$ ) it is necessary to compute $A h^{*}\left(t_{\mathrm{AP}}\right)$ and indirectly $\Delta A h\left(t_{\mathrm{RP}}\right)$.

Based on what already described, and thanks to Eq. (18), the set of equation allowing for the $C_{\mathrm{SC}}$ computation are:

$C_{\mathrm{SC}}\left(t_{\mathrm{RP}}\right)=C_{\mathrm{SC}}^{*}\left(t_{0}\right)+\Delta C_{\mathrm{SC}}\left(t_{\mathrm{RP}}\right) t_{0}<t<t_{\mathrm{RP}}$

$C_{\mathrm{SC}}^{*}(t)=F \mathrm{e}^{-f\left(A h^{*}\left(t_{\mathrm{RP}}\right)+A h(t)\right)}+K \mathrm{e}^{-k\left(A h^{*}\left(t_{\mathrm{RP}}\right)+A h(t)\right)}+C_{\mathrm{PC} \infty} t>t_{\mathrm{RP}}$

$A h^{*}\left(t_{\mathrm{RP}}\right) \rightarrow F \mathrm{e}^{-f A h^{*}\left(t_{\mathrm{RP}}\right)}+F \mathrm{e}^{-f A h^{*}\left(t_{\mathrm{RP}}\right)}+C_{\mathrm{PC} \infty}=C_{\mathrm{SC}}\left(t_{\mathrm{RP}}\right)$

The value of $A h^{*}\left(t_{\mathrm{RP}}\right)$ satisfying Eq. (23) is computed via the inverse function of Eq. (3). ${ }^{6}$

Before showing the experimental validation of the proposed model, we would like to underline the following points:

- the recovering and accelerated ageing phases appear only at the time indicated in Eqs. (6)-(9);

- if the transition has a duration shorter than the ones specified by Eqs. (6)-(9), the associated recovering or accelerated ageing effect does not exist;

- if the transition has longer duration shorter than the ones specified by Eqs. (6)-(9), the associated recovering or accelerated effect are compensated by the normal ageing mechanism driving the performance fading in the targeted LE or PC test.

\section{Experimental validation}

This section aims at experimentally validating the model described in Section 3. To this purpose we have adopted two different combined LE and PC stress conditions. It is important to underline that:

- the SCs used for these validations do not belong to the same batch production of the one used for the parameter assessment, neither for LE and PC mapping and neither for AP and RP effects evaluation;

- the duration of each sub-phases is different from the LE and PC stress used for the $\mathrm{SOH}$ model prediction parameter assessment.

The current profile used during the PC stress conditions is shown in Fig. 4.

Fig. 5 illustrates the $C_{\mathrm{SC}}$ evolution during a combined LE and PC stress at $T_{\mathrm{SC}}$ equal to $328.15 \mathrm{~K}$. This specific cycle starts with a LE

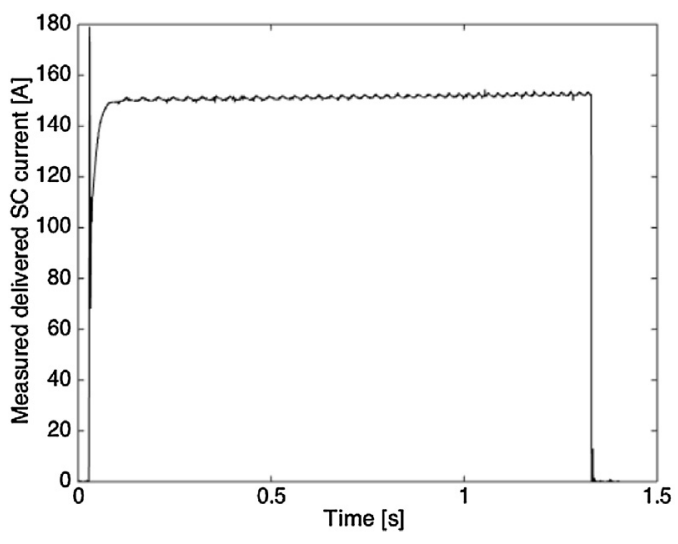

Fig. 4. measured high-pulsed current profile adopted for the SC PC stress. 


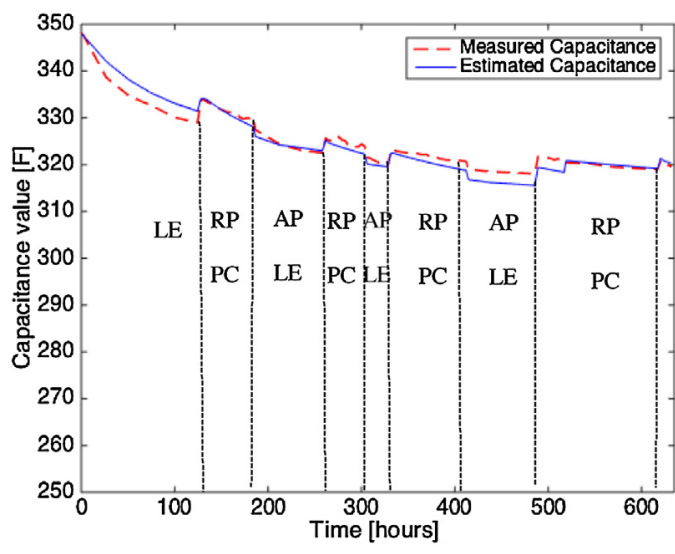

Fig. 5. Evolution of $C_{S C}$ during a combined $P C$ and LE ageing stress at $328.15 \mathrm{~K}$ (first case): comparison between measured and estimated $\mathrm{C}_{\mathrm{SC}}$.

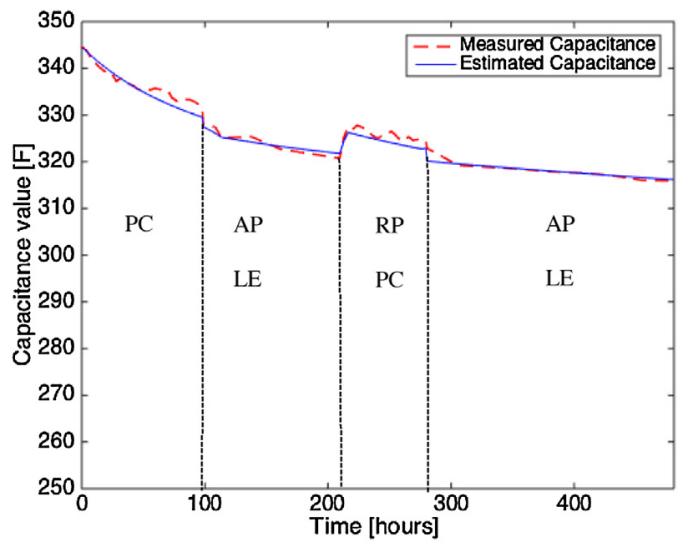

Fig. 6. Evolution of $C_{S C}$ during a combined PC and LE ageing stress at $328.15 \mathrm{~K}$ (second case): comparison betwee measured and estimated $\mathrm{C}_{\mathrm{SC}}$.

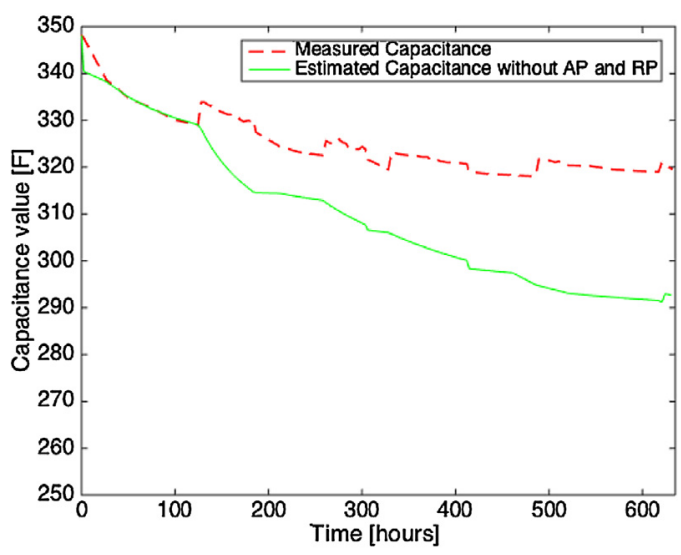

Fig. 7. Evolution of $C_{S C}$ during a combined PC and LE ageing stress at $328.15 \mathrm{~K}$ (first case) without taking into account accelerated ageing phase (AP) and recovering phase (RP).

stress and it finishes with a PC one. Its duration is of 35 days and it is composed by 12 transitions LE toward PC or PC toward LE stress.

Fig. 6 illustrates the $C_{\mathrm{SC}}$ evolution during a combined LE and PC stress at $T_{\mathrm{SC}}$ equal to $328.15 \mathrm{~K}$ having a different composition of PC and LE durations. It starts with a PC stress and it finishes with a LE stress. The numbers of transitions from different types of stress is

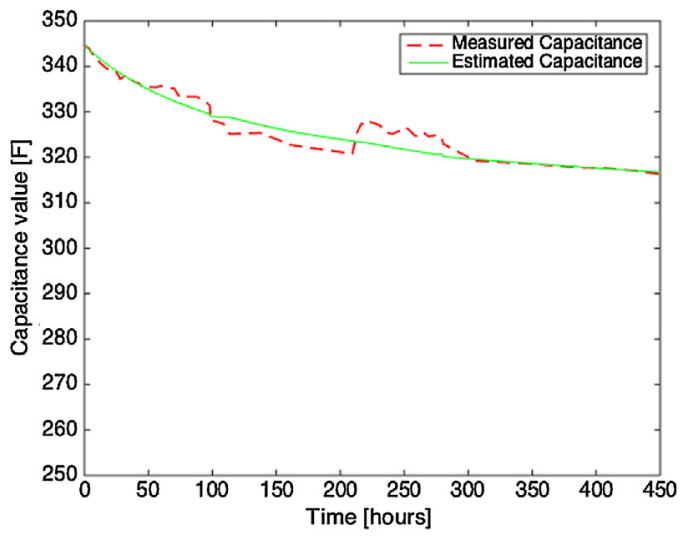

Fig. 8. Evolution of $\mathrm{C}_{\mathrm{SC}}$ during a combined PC and LE ageing stress at $328.15 \mathrm{~K}$ (second case) without taking into account accelerated ageing phase (AP) and recovering phase (RP).

limited to 5. Its duration is of 22 days. In both figures the experimentally inferred $C_{\mathrm{SC}}$ and the assessed one are reported.

By observing these results it is possible to conclude that the proposed $\mathrm{SOH}$ model is able to accurately predict the evolution of $C_{\mathrm{SC}}$. The root mean square (RMS) is below 1.74 for Fig. 5 and below 1.3 for Fig. 6.

The relative error at the end of the targeted cycle is of $0.15 \%$ and $0.12 \%$. The maximum error is of $0.97 \%$ and $0.81 \%$, for the twotargeted stress respectively. ${ }^{9}$

In order to assess the influence of the AP and RP on the SOH performances, we here report in Figs. 7 and 8 the $C_{\mathrm{SC}}$ evolution without accounting for these sub-phases. The measured $C_{\mathrm{SC}}$ are the same used in Figs. 5 and 6.

For the first cycle, it is evident the importance to take into account the effects of the AP and the RP, since at each transition LE to PC (and vice versa) there is an important cumulative error leading to a final relative error of $9.2 \%$. For the second cycle, since the number of transitions from LE toward PC stress is lower than the first case, the maximum error is of $4.6 \%$.

In any case the absence of the correct representation of these sub-phases is of crucial importance for an accurate $\mathrm{SOH}$ computation.

\section{Conclusions}

The work here presented has illustrated a model for the $\mathrm{SOH}$ prediction of electrochemical double layer SCs.

Based on the findings of part I, this model refers to the prediction of the SC capacitance $C_{\mathrm{SC}}$. The structure of the proposed SOH model makes use of the phenomenological description of the SC ageing processes given in part I where PC and LE ageing stresses have been considered to study the SC ageing.

Two main blocks compose the model. The first one refers to the mapping of the evolution of the $C_{\mathrm{SC}}$ during reference LE and PC stresses. This mapping is realised by two exponential functions whose parameters are inferred via a least square fitting. The second block of the model modifies the inputs given to the first one in order to adapt the stressing conditions different from the reference ones.

\footnotetext{
${ }^{9}$ The mean root square error adopted to quantify the performances of the proposed $\mathrm{SOH}$ model is defined as follows:

$\sum_{i=1}^{N} \sqrt{\left[\left(C_{\text {SCmea }, i}-C_{\mathrm{SC}, i}^{*}\right)^{2}\right]} / N$,

where $C_{\mathrm{SCmea}}$ is the measured value of capacitance and $N$ the number of tagged points for the error computation.
} 
Table 3

Experimental evaluation of the solvent losses in SC during PC test.

\begin{tabular}{llll}
\hline $\begin{array}{l}\text { Average } \\
\text { initial Weight }\end{array}$ & $\begin{array}{l}\text { Average } \\
\text { final Weight }\end{array}$ & Absolute solvent losses \\
\hline $146.3620 \mathrm{~g}(328.15 \mathrm{~K})$ & $146.3031 \mathrm{~g}$ & $58.9 \mathrm{mg}$ & $0.29 \%$ \\
$148.5465 \mathrm{~g}(308.15 \mathrm{~K})$ & $148.4933 \mathrm{~g}$ & $53.2 \mathrm{mg}$ & $0.25 \%$ \\
\hline
\end{tabular}

In summary, the main features of the proposed model are: (i) its ability to assess the $C_{\mathrm{SC}}$ evolution during LE and PC stresses characterised by durations different from the reference ones and (ii) its capability to account for the effects of AP and RP.

The proposed $\mathrm{SOH}$ model has been validated via experimental tests on a $365 \mathrm{~F}$ supercapacitor stressed with LE and PC conditions other than the ones adopted to infer the model parameters. The results have shown a relative error between measurement and prediction below $0.8 \%$. Additionally, this experimental validation has demonstrated that the absence of the AP and RP in the SOH model might produce a large misestimating of the SC SOH.

\section{Appendix A.}

\section{Solvent evaporation}

This section aims at discussing the experimental evidences on the solvent evaporation described in part I.

As it has been illustrated in the first part of this work, one of the main reasons associated to the SC capacitance fading could be the solvent evaporation. Indeed this involves a reduction of the available charge inside the SC. In order to experimentally justify what illustrated in the first part of this manuscript, the weight of two targeted SCs aged with a PC stress at $328.15 \mathrm{~K}$ and at $308.15 \mathrm{~K}$ respectively during 2 weeks has been measured before and after the test.

The measurement of the SC weight has been done with a Metler Toledo XS205 balance. The available weight range of this device is from 0 up to $220 \mathrm{~g}$ with an accuracy of $\pm 0.1 \mathrm{mg}$.

Table 3 summarizes the weight of the two targeted SCs before and after the test. The average values have been computed as mean value of 5 weight measurements. In Table III it is possible to observe a relative solvent loss around $0.3 \%$ of the initial electrolyte amount. It is worth nothing that a quota of the evaporated solvent it still inside the SC and consequently the value of relative solvent loss should be higher. It is important to underline that the relative solvent evaporation is less important for the test performed at the lower temperature. This is in agreement with the explanation given in part I of this manuscript. In any case, these measurements allowed for a computation of the solvent evaporation that could partially justifies the $C_{\mathrm{SC}}$ fading of $13 \%$ during the first two weeks of combined PC and LE stresses.

\section{References}

[1] E. Bilbao, P. Barrade, I. Etxeberria-Otadui, A. Rufer, S. Luri, I. Gil, Optimal energy management strategy of an improved elevator with energy storage capacity based on dynamic programming, IEEE Trans. Ind. Appl. 50 (2) (2015) 1233-1244 March-April 2014.

[2] N. Mendis, K.M. Muttaqi, S. Perera, Management of low- and high-frequency power components in demand-generation fluctuations of a DFIG-based winddominated RAPS system using hybrid energy storage, IEEE Trans. Ind. Appl. 50 (3) (2014) 2258-2268 May-June.

[3] A. Nasiri, O. Abdel-Baqi, P. Miller, Dynamic performance improvement and peak power limiting using ultracapacitor storage system for hydraulic mining shovels, IEEE Trans. Ind. Appl. 90.

[4] M. Ristic, Y. Gryska, J.V.M. McGinley, V. Yufit, Supercapacitor energy storage for magnetic resonance imaging systems, IEEE Trans. Ind. Electron. 61 (8) (2014) 4255-4264 August.

[5] P. Kreczanik, P. Venet, A. Hijazi, G. Clerc, Study of supercapacitor aging and lifetime estimation according to voltage, temperature, and RMS current, IEEE Trans. Ind. Electron. 61 (9) (2014) 4895-4902 September.

[6] Oliver Bohlen, Julia Kowal, Dirk Uwe Sauer, Ageing behaviour of electrochemical double layer capacitors. Part I: experimental study and ageing model, J. Power Sources 172 (2007) 468-475.

[7] Oliver Bohlen, Julia Kowal, Dirk Uwe Sauer, Ageing behaviour of electrochemical double layer capacitors. Part II: lifetime simulation model for dynamic applications, J. Power Sources 173 (2007) 626-632.

[8] A. Hammar, P. Venet, R. Lallemand, G. Coquery, G. Rojat, Study of accelerated aging of supercapacitors for transport applications, IEEE Trans. Ind. Electron. 57 (12) (2010) 3972-3979 December.

[9] O. Briat, J.-M. Vinassa, N. Bertrand, H. El Brouji, E. Woirgard, Impact of calendar life and cycling ageing on supercapacitor performance, IEEE Trans. Veh. Technol. 58 (8) (2009) 3917-3929 October.

[10] S.N. Motapon, L.-A. Dessaint, K. Al-Haddad, A comparative study of energy management schemes for a fuel-cell hybrid emergency power system of moreelectric aircraft, IEEE Trans. Ind. Electron. 61 (3) (2014) 1320-1334 March.

[11] V. Lystianingrum, V.G. Agelidis, B. Hredzak, State of health and life estimation methods for supercapacitors, Power Engineering Conference (AUPEC), Australasian Universities, 2013, pp. 1-7 September 29, 2013-October 3, 2013.

[12] Dave Andre, Christian Appel, Thomas Soczka-Guth, Dirk Uwe Sauer, Advanced mathematical methods of SOC and SOH estimation for lithium-ion batteries, J. Power Sources 224 (2013) 20-27.

[13] Scott J. Moura, Nalin A. Chaturbedi, Miroslav Krstić, Adaptive partial differential equation observer for battery state-of-charge/state-of-health estimation via an electrochemical model, J. Dyn. Syst. Meas. Contr. 136 (1) (2013) 011015 October 15

[14] Dimitri Torregrossa, Kathryn E. Toghill, Hubert H. Girault, Mario Paolone, Understanding the ageing process, recovering phase and fault diagnosis of electrochemical double layer capacitors, IEEE Sustainable Technology Conference, 24-26 July, 2014.

[15] D. Torregrossa, K.E. Toghill, V. Amstutz, H.G. Hubert, M.P. irault, aolone, Macroscopic indicators of fault diagnosis and ageing in electrochemical double layer capacitors, J. Energy Storage 2 (2015) 8-24 August.

[16] D. Torregrossa, M. Paolone, Novel experimental investigation of supercapacitor ageing during combined life-endurance and power-cycling tests, IECON (2013) November.

[17] Makato Ue, Yukio Sasaki, Yasutaka Tanaka, Masayuki. Morita, Nonaqueous electrolytes with advances in solvents, Electrolytes for Lithium and Lithiumion Batteries, Springer, New York, 2014 (Chapter 2).

[18] Zongli Dou, Rong Xu, Alfonso Berduque, The Development of Electrolytes in Aluminium Electrolytic Capacitors for Automotive and High Temperature Applications Research and Development Department, BHC Components LTD (KEMET).

[19] Mouad Dahbi, Fouad Ghamouss, Francois Tran-Van, Daniel Lemordant, Mérièm Anouti, Comparative study of EC/DMC LiTFSI and LiPF6 electrolytes for electrochemical storage, J. Power Sources 196 (22) (2011) 9743-9750 15 November. 